\title{
Microwave imaging for stroke detection: validation on head- mimicking phantom
}

\author{
Behnaz Sohani ${ }^{1}$, Gianluigi Tiberi ${ }^{1}$, Navid Ghavami ${ }^{2}$, Mohammad Ghavami ${ }^{1}$, Sandra Dudley ${ }^{1}$ Amir \\ Rahmani $^{3}$ \\ ${ }^{1}$ School of Engineering, London South Bank University, London, UK, \\ ${ }^{2}$ UBT - Umbria Bioengineering Technologies, Spin off of University of Perugia, Perugia, Italy, \\ ${ }^{3}$ Castlet Ltd, Lincoln, UK.
}

\begin{abstract}
This paper provides initial results on the efficacy of Huygens Principle (HP) microwave imaging for haemorrhagic stroke detection. This is done using both simulations and measurements in an anechoic chamber. Microstrip antennas operating between 1 and $2 \mathrm{GHz}$ have been designed, constructed and used for imaging a human head model in Computer Simulation Technology (CST) software. A 3D model consisting of human head tissues of Ella is employed in the simulation. An emulated haemorrhagic stroke with the dielectric properties equivalent to the blood has been inserted in Ella. Moreover, a 3-layered head-mimicking phantom containing an inclusion has been constructed. Frequency-domain measurements have been performed in an anechoic chamber using a Vector Network Analyser arrangement to obtain the transfer function (S21) between two antennas. Both simulations and measurements show that the HP based technique may be used for haemorrhagic stroke detection. Among linear scattering techniques, the HP based technique allows to detect dielectric inhomogeneities in the frequency domain. HP can also be used if the antennas and phantom are in free space, i.e. no coupling liquid is required. Detection of the haemorrhagic stroke has been achieved after removing the artefacts. Artefact removal is an essential step of any microwave imaging system and current artefact removal approaches have been shown to be ineffective in the specific scenario of brain imaging. However, one of this paper's novel contributions is the proposal of an artefact removal algorithm based on a subtraction between S21 obtained using measurements, which achieves improved performance while having a much lower computational complexity.
\end{abstract}

\section{INTRODUCTION}

Strokes are the leading cause of adult disability and are the number two cause of death worldwide [1]. A stroke can be cast into two major categories, ischemic and haemorrhagic [2]. A haemorrhagic stroke occurs when a blood vessel bursts inside the brain; the blood accumulates and compresses the surrounding brain tissue. The course of treatment for ischemic and haemorrhagic strokes is different [3]. An initial examination done by a physician is typically via a neurological exam. This however is not always conclusive, as many non-vascular conditions can simulate stroke symptoms [4]. As such, physicians primarily rely on medical imaging systems such as the computed tomography (CT) scan and magnetic resonance imaging (MRI) for confirmation of the diagnosis. The complexity of stroke diagnosis emphasizes the vital importance played by the CT and MRI scan systems. They are however, not fast, cost effective or portable, nor are they accessible at rural medical clinics, or carried by first response paramedical teams. Microwave imaging to detect brain abnormalities has recently been proposed [5-7]. Diagnostic microwave imaging exploits the differences between tissues' dielectric properties and it has been demonstrated that brain-stroke changes the dielectric properties of the tissues. Haemorrhagic stroke leads to a significant increase in the dielectric properties (up to 20\%) with respect to the dielectric properties of the white/grey matter [2]. Potentially, microwave imaging can supplement current diagnostic methods as it may potentially provide a fast, cost-effective and portable detection system [6]. Specifically, in recent years, there has been a growing interest in the development of imaging methods such as those using the microwave imaging technique for medical applications. Its use of nonionizing signals, low-cost, low complexity and its ability to penetrate through mediums are some of the benefits of microwave technology [8]. As already mentioned, microwave imaging applied to the brain could have great potential in enabling non-ionizing, transportable devices, particularly emergency or mobile situations. Current research in microwave imaging can be divided mainly into tomography and linear scattering techniques. The tissue reconstruction using microwave tomography has limitations as it requires the solution of a complicated mathematical inverse problem. In this paper we focus on the 
other approach, quite similar to radar imaging, which has the objective to localise strong scatterers. In contrast to tomography, our approach does not reconstruct the dielectric properties inside the domain, but investigates the presence of the main scattering centre. The microwave radar approach solves a simpler computational problem by seeking only to identity the significant scatters. Among linear scattering techniques, the HP based technique allows to detect dielectrics inhomogeneities in frequency domain [6]. In addition, HP requires a very simple hardware set-up, i.e. one transmitting antenna and one receiving antenna (coupled through a Vector Network Analyser) which rotate around the object to collect the signals in a multi-biostatic fashion. Up to now, HP has been used for breast cancer and skin cancer detection. The aim of this paper is to provide initial results on the efficacy of HP microwave imaging for haemorrhagic stroke detection. This is done using both simulations and measurements in an anechoic chamber. This paper is organized as follows: section 2 explains the antenna design and simulation. In section 3, the measurements are demonstrated. Results are discussed in section 4, and finally section 5 concludes the paper.

\section{ANTENNA DESIGN AND SIMULATION}

The main focus of this section is the design of a wideband (WB) microstrip antenna operating between 1 and 2 GHz. This band has shown to be optimal from brain imaging [9]; in addition, in [10] it is clearly highlighted that WB can enhance performance in lesion detection. To obtain enhancement in bandwidth, an isosceles triangular patch antenna using a new technique which is fractal ground plane, has been designed. The initial idea of using fractal ground plane technique has been adopted from [11]. The fabricated triangular patch antenna with fractal ground plane is shown in Fig 1.

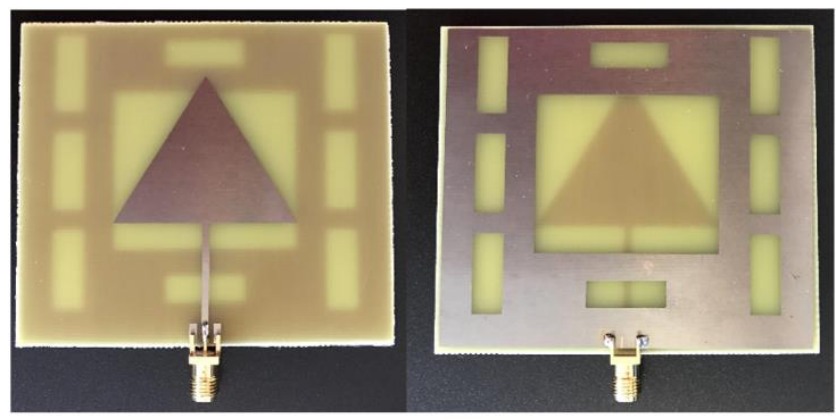

(a)

(b)

Fig 1. Fabricated triangular patch microstrip antenna with fractal ground plane: (a) top view (b) bottom view.

The antenna is designed using FR-4 substrate material with a dielectric constant of $\varepsilon_{\mathrm{r}}=4.7$. The input power for the discrete edge port is $1 \mathrm{Watt}$ (peak). The patch geometry is isosceles triangular shape. The details of the geometry are illustrated in Fig 2. This antenna has a single-layer structure and Fig 2 (a) shows the front view of triangular microstrip patch antenna. There are four different parts: triangular patch and microstrip feed line (at the top), substrate (in the middle) and fractal ground plane (at the bottom).

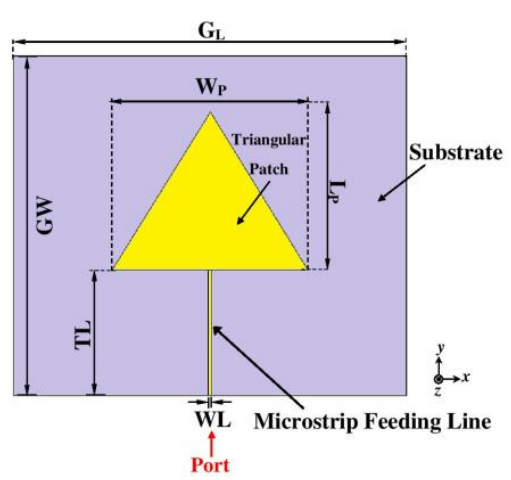

(a)

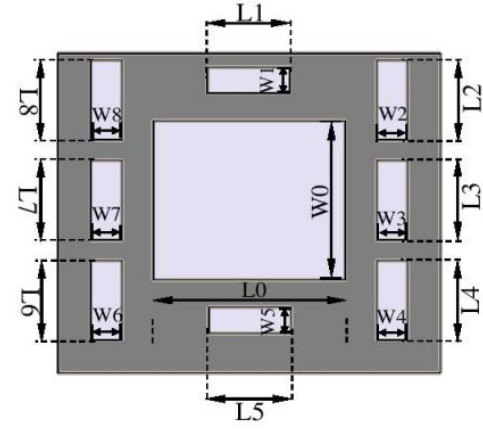

(b)

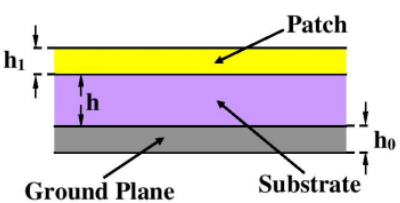

(c)

Fig 2. Geometry and dimensions of the proposed antenna: (a) front view (Patch and substrate), (b) back view (Fractal ground plane), and (c) side view of the overall structure. 
Dimensions of the patch can be calculated to excite the first resonant frequency by applying equations used in [12], [13], and [14] as follows:

- Width of patch $\left(W_{P}\right)$ :

$$
W_{P}=\frac{c}{2 f_{0} \sqrt[2]{\frac{\varepsilon_{r}+1}{2}}}
$$

- Actual length of patch $\left(L_{P}\right)$ :

$L_{P}=L_{\text {eff }}-2 \Delta L$

Where:

$$
\begin{aligned}
& L_{\text {eff }}=\frac{C}{2 f_{0} \sqrt[2]{\varepsilon_{e f f}}} \\
& \Delta L=0 \cdot 412 h \frac{\left(\varepsilon_{e f f}+0 \cdot 3\right)\left(\frac{W_{P}}{h}+0 \cdot 264\right)}{\left(\varepsilon_{e f f}-0 \cdot 264\right)\left(\frac{W_{P}}{h}+0 \cdot 8\right)}
\end{aligned}
$$

- Length of the microstrip transmission line $(T L)$ :

$T L=\frac{\lambda}{4}=\frac{\lambda_{0}}{4 \sqrt{\varepsilon_{r}}}$

With:

$$
\lambda_{0}=\frac{c}{f_{0}}
$$

In the above equations, the subscript eff indicates the effective values of the parameters. The computed values of $W_{P}, L_{P}$ and $T L$ are $39.5 \mathrm{~mm}, 31.5 \mathrm{~mm}$ and $16.8 \mathrm{~mm}$, respectively. The overall dimension of the substrate and ground plane is $G_{W} \times G_{L} \times h$. The length and width of the patch are $L_{P}$ and $W_{P}$, as shown in Fig 2 (a). In Fig 2 (b), we can see the fractal ground plane, which consists of nine rectangles with different sizes. A fractal ground can be appropriately designed to ensure WB requirements [15]. The dimensions of the slots in the fractal ground plane are labelled by $L$ and $W$. The $50 \Omega$ discrete microstrip feeding structure is used at the bottom part of the patch. By optimizing the dimensions and shapes of the slots in the ground structure and the triangular antenna structure, the impedance bandwidth of this antenna can be clearly improved. The front view of the proposed antenna with the fractal ground plane is shown in Fig 2 (a) and all the optimized dimensions of the proposed antenna are illustrated by Table I.

TABLE I

OPTIMIZED DIMENSIONS OF THE PROPOSED ANTENNA

(Unit: $\mathrm{mm}$ ).

\begin{tabular}{|c|c|c|c|c|c|c|}
\hline$W_{P}=39.5$ & $W_{L}=0.66$ & $T L=16.8$ & $G_{L}=79$ & $G_{W}=68.28$ & $L_{P}=31.5$ & $h=1.2$ \\
\hline$h_{0}=0.035$ & $h_{l}=0.035$ & $L_{0}=39.50$ & $L_{(1, \ldots, 8)}=17.07$ & $W_{0}=34.14$ & $W_{(2,3,4,6,7,8)}=6.61$ & $W_{(1,5)}=5.69$ \\
\hline
\end{tabular}

The proposed antenna is simulated in both free space and near a human head model using CST microwave studio software. Specifically, a three-dimensional (3D) model consisting of human head tissues of the Ella model (ITIS Foundation, Switzerland) is employed with segmentation of $2 \times 2 \times 2$ $\mathrm{mm}^{3}$, as shown in Fig 3. The Ella model provides details of electric conductivity and relative permittivity for the different tissues of a female at the age of 26 [16]. An emulated haemorrhagic stroke, having dimensions of a spherical object with radius of $10 \mathrm{~mm}$, has been inserted in Ella. The dielectric properties of the emulated haemorrhagic stroke are equivalent to blood. The transmitting antenna has been placed $17 \mathrm{~cm}$ away from the centre of the Ella model's head.

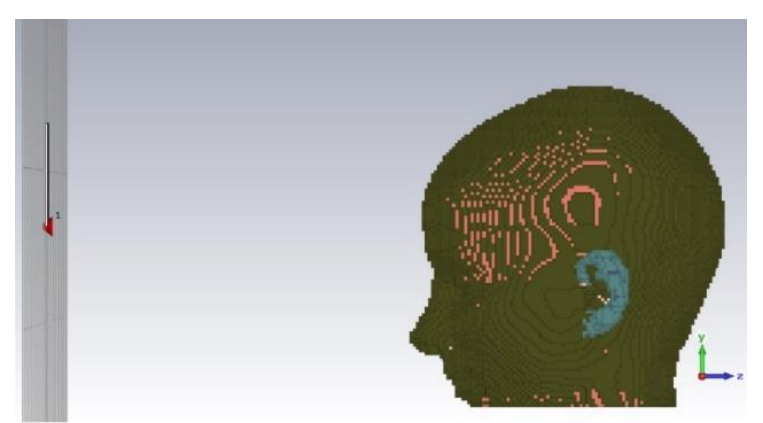

Fig 3. The proposed antenna and 3D model of human head using CST software. 
The proposed triangular antenna has also been fabricated in-house at LSBU (as shown in Fig 1), and the correspondent $S_{11}$ measurements have been taken in an anechoic chamber, both in free-space and in front of a human head using a Vector Network Analyser (Anritsu MS2028C VNA Master manufactured by Anritsu EMEA Ltd). Fig 4 shows the simulated and measured scatter parameter $S_{11}$ for the proposed antenna both in free space and near the head. Looking at Fig 4 (a), the operational frequency is $1.6 \mathrm{GHz}$ with a bandwidth $>10 \%$. Fig 4(b) displays the measured and simulated $S_{11}$ of the proposed antenna in front of human head.

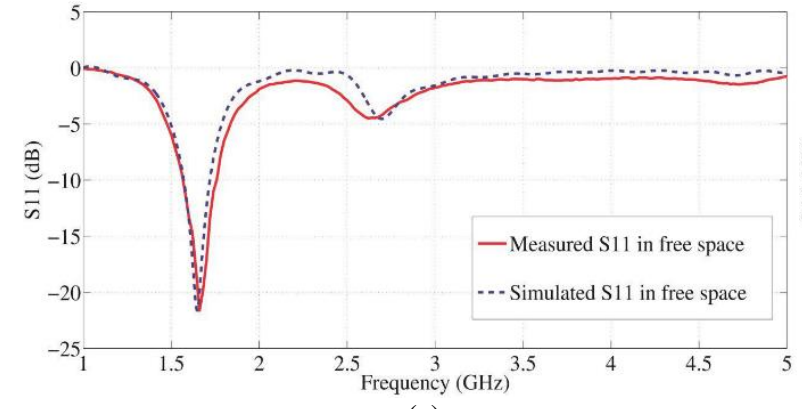

(a)

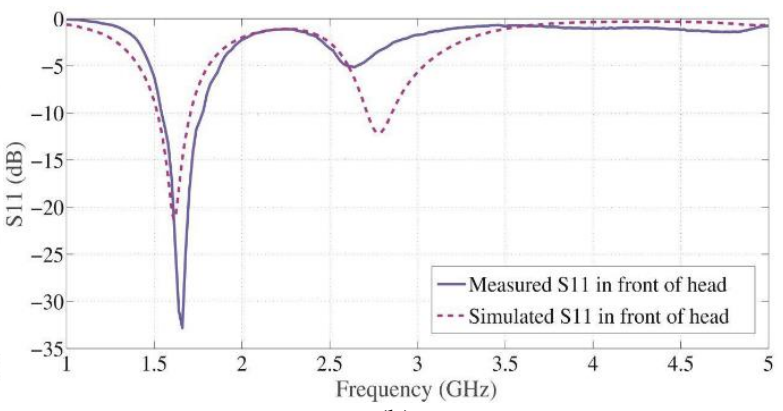

(b)

Fig 4. Measured and simulated S11 for the patch antenna: (a) in free space and (b) in front of human head.

As shown in Fig 4, the measured $S_{11}$ both in free space and in front of human head shows excellent agreement with the simulation results. In addition, measurements reveal that matching and bandwidth slightly increase in the presence of the human head. Furthermore, an operational bandwidth greater than $10 \%$ cantered at $1.6 \mathrm{GHz}$ makes this antenna an ideal candidate for brain imaging experiments. In fact, in [6] it has been shown that higher frequencies are not suitable for brain imaging due to the high signal attenuation, while lower frequencies will decrease resolution [10]. Figure 5 Shows the magnitude of ycomponent of the electric field for; (a) Ella model, (b) when the emulated stroke is included in the Ella model and (c) the differences of the Ella model and the Ella model with stroke. Moreover, the ycomponent of the electric field on one external surface of the head is calculated and used for performing the imaging, as shown in section 4. Specifically, the electric field is calculated on a circular grid of points with radius of $110 \mathrm{~mm}$ and $6^{\circ}$ phi sampling just outside the Ella head model.

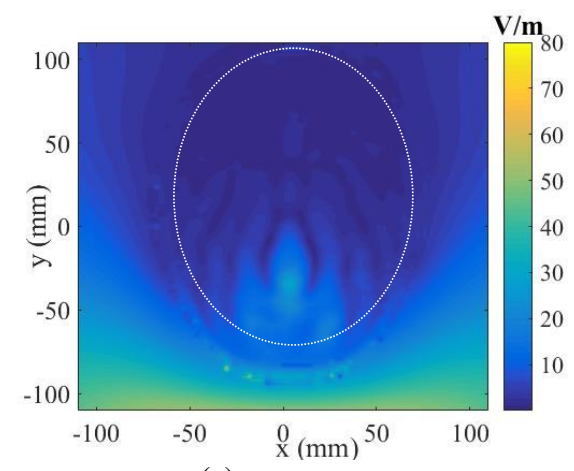

(a)

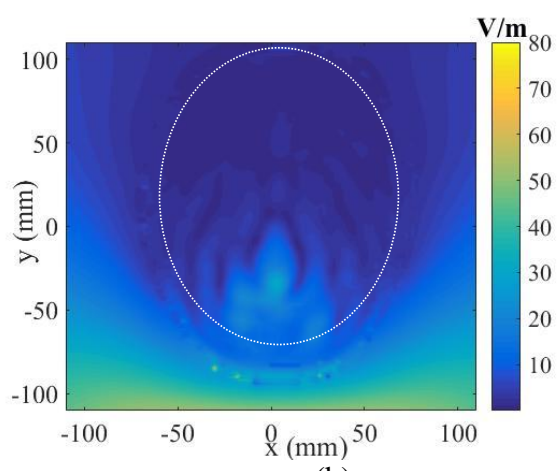

(b)

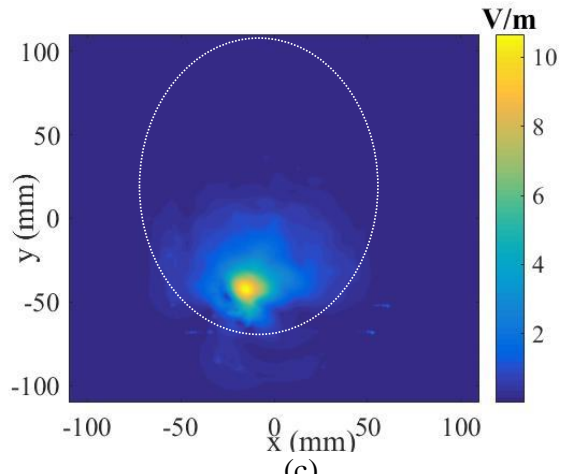

(c)

Fig 5. The electric field for; (a) the Ella model, (b) when the emulated stroke is included in the Ella model and (c) the differences of the Ella model and the Ella model with stroke. 


\section{MEASUREMENTS \\ 3.1 Phantom Fabrication}

The phantom presented in this paper, used for microwave imaging experiments over an operating frequency ranging from $1 \mathrm{GHz}$ to $2 \mathrm{GHz}$, has been designed as a multilayer phantom with each layer corresponding to a particular part of the human head. To allow feasible manufacturing, our proposed phantom has been constructed using three layers, each of which mimics: I) Skull, II) a combination of grey matter and white matter, III) Blood inclusion. The dielectric constant of each layer is given in Table II, where the values have been derived from [17], [18], and [19]. A specific recipe has been considered for each layer with the aim of simulating dielectric parameters of human tissues.

TABLE II

DIELECTRIC CONSTANT AND CONDUCTIVITY AT THE FREQUENCY OF 1.6 GHZ [17], [2] and [18].

\begin{tabular}{|c|c|c|}
\hline Tissue & Relative Permittivity(E) & 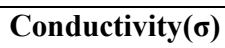 \\
\hline $\begin{array}{l}\text { Realistic human brain (combination of grey } \\
\text { matter and white matter) }\end{array}$ & 38 & $0.59 \mathrm{~S} / \mathrm{m}$ \\
\hline Skull & 10 & $0.96 \mathrm{~S} / \mathrm{m}$ \\
\hline Blood & 61 & $1.58 \mathrm{~S} / \mathrm{m}$ \\
\hline
\end{tabular}

As Fig 6 and Table III show, three different volumes of container have been used for constructing the phantom and then they have been filled up with different specific materials to form the layers. Constructing the phantom has been performed through the following steps. The small cylindrically shaped container has been filled up with blood equivalent material.

The brain mould has been filled with grey matter and white matter equivalent material. Then both the blood container (which is situated inside the brain mould) and the brain mould are subsequently placed in the large plastic container. In this step, skull equivalent materials have been poured to completely fill the remaining space surrounding the brain and the blood, as shown in Fig 7. Each layer of the phantom has been constructed by mixing constituent materials with the appropriate ratio. Skull has been designed to be solid, while the combination of grey matter and white matter and the blood have been designed as liquid.

TABLE III

PHANTOM LAYERS' DESIGN HEIGHT AND SIZE

\begin{tabular}{|c|c|c|c|}
\hline $\begin{array}{c}\text { Tissue layers of the } \\
\text { phantom }\end{array}$ & $\begin{array}{c}\text { Length } \\
(\mathbf{c m})\end{array}$ & $\begin{array}{c}\text { Width } \\
(\mathbf{c m})\end{array}$ & $\begin{array}{c}\text { Height } \\
(\mathbf{c m})\end{array}$ \\
\hline Skull & 21 & 19.5 & 12 \\
\hline $\begin{array}{c}\text { Grey matter and } \\
\text { white matter }\end{array}$ & 19 & 16.5 & 9 \\
\hline Blood & 10 & 10 & 4.5 \\
\hline
\end{tabular}

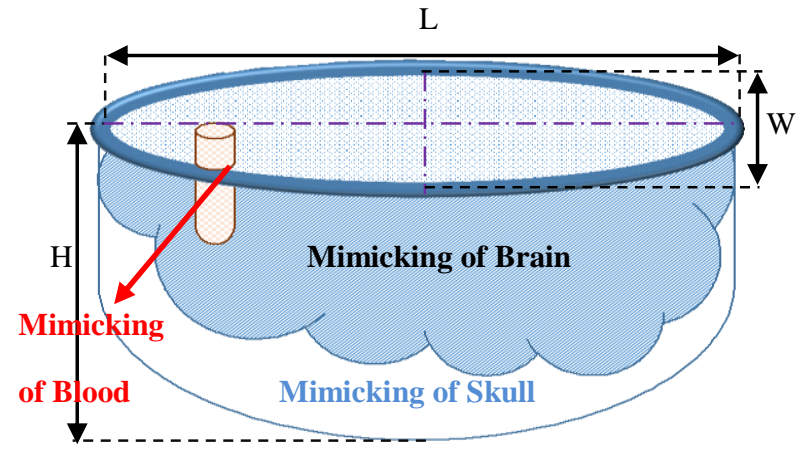

Fig 6. Design of different layers of phantom.

The ingredients of the recipe for mimicking the skull tissue have been shown in table IV [19]. 
TABLE IV

TISSUE MIMICKING RECIPES.

\begin{tabular}{|c|c|}
\hline Ingredient (Grams) & Skull \\
\hline Flour & 55 \\
\hline Oil & 35 \\
\hline Salt & 0.3 \\
\hline Sugar & 11 \\
\hline Deionized water & 15 \\
\hline
\end{tabular}

The details of the proposed phantom fabrication method are as follows.

I. Skull mimicking tissue:

- First, mix the flour and oil in a mixing bowl together and beat into a smooth batter. Stir until you no longer see dry clumps of flour.

- Then, mix deionized water with sugar and stir until the sugar was completely dissolved in the water.

- Combine the two mentioned mixes until it formed a uniform shape.

II. Brain mimicking tissue:

- In the mixing bowl, stir a mixture of glycerol and deionized water with ratios of $40 \%$ and $60 \%$, respectively as given in [20].

III. Blood mimicking tissue:

- The blood has been mimicked using a mixture of glycerol and deionized water with ratios of $15 \%$ and $85 \%$, respectively as given in [20].

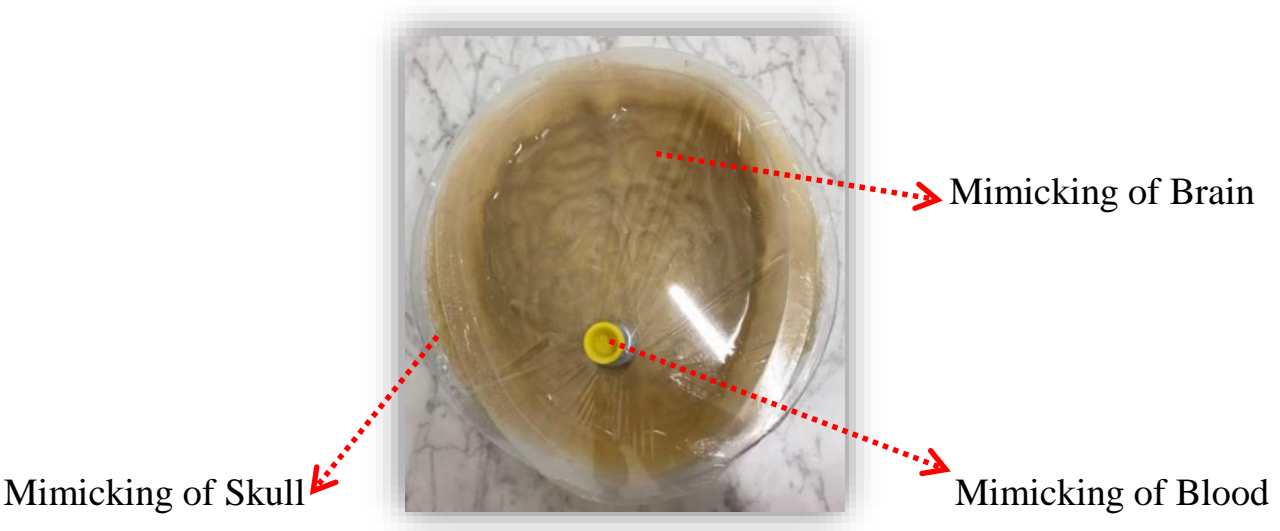

Fig 7. The fabricated human head phantom.

\subsection{Measurements in an Anechoic Chamber}

Frequency-domain measurements have been performed in an anechoic chamber using a VNA arrangement to obtain the transfer function (S21) between the two antennas. The fabricated antennas vertically polarized were used, after calibration. The phantom was placed in the centre of a rotatable table. In Fig 8, the fabricated phantom has been shown inside the anechoic chamber. In order to exploit the variation of signals at different frequencies, the complex S21 values were recorded over a wide frequency range of 1-2 GHz, using a frequency sample spacing of $5 \mathrm{MHz}$. For each set of measurements, the transmitting antenna has been placed $17 \mathrm{~cm}$ away from the centre of the rotating table. For each transmitting position, we recorded the $\mathrm{S} 21$ at $N_{P T}=60$ receiving positions. Specifically, we measure S21 at the points $\mathrm{rx}_{\mathrm{np}} \equiv\left(\mathrm{a}_{0} ; \phi_{\mathrm{np}}\right) \equiv \vec{\rho}_{\mathrm{np}}$, displaced along a circular surface having radius $\mathrm{a}_{0}$. To be exact, $\mathrm{a}_{0}$ is the distance between the centre of the rotating table and receiving antenna which is $14 \mathrm{~cm}$ [21]: 
$\left.S 21_{\mathrm{tx}_{\mathrm{m}}}^{\mathrm{known}}\right|_{\mathrm{rx} \mathrm{np}_{\mathrm{p}}}=S 21_{\mathrm{np}, \mathrm{tx} \mathrm{m}}^{\text {known }} \quad$ With $n p=1, \ldots, N_{P T}$.

$m$ is the transmitting position and $n p$ is the receiving position. The procedure has been repeated considering two transmitting positions, displaced $5^{\circ}$ from each other. Note that, rather than moving the transmitting antenna, the two transmitting positions have been synthetized by appropriately rotating the phantom.

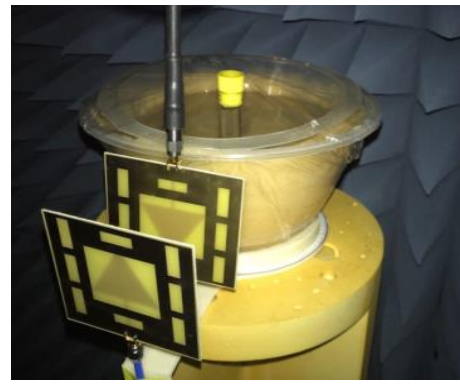

Fig 8. Position of the phantom inside anechoic chamber.

\section{IMAGING PROCEDURE AND RESULTS}

We perform imaging of the head phantom as superposition of the fields radiated by the $N_{P T}$ receiving points of eq. (7):

$E_{\mathrm{HP}, 2 \mathrm{D}}^{\mathrm{rcstr}}\left(\rho, \emptyset ; \mathrm{tx}_{\mathrm{m}} ; f\right)=\Delta_{S} \sum_{n p=1}^{N_{P T}} S 21_{\mathrm{np}, \mathrm{tx}_{\mathrm{m}}}^{\mathrm{known}} G\left(k_{0}\left|\overrightarrow{\rho_{n p}}-\vec{\rho}\right|\right)$

Where $\vec{\rho} \equiv(\rho, \emptyset)$ is the observation point; $\mathrm{k}_{0}$ represents the wave number in free space (due to the presence of receiving antenna in free space); and $\Delta_{S}$ is the spatial sampling; $E_{\mathrm{HP}, 2 \mathrm{D}}^{\mathrm{rcstr}}$ stands for the "reconstructed" internal electric field. Moreover, it is noted that the reconstructed electric field depends on the illuminating source and frequency. In eq. (8), the Green's function $G$ is used to propagate the field. Suppose that we use $\mathrm{N}_{\mathrm{F}}$ frequencies, then the intensity of the final image $(I)$ can be obtained through the following equation, i.e. by adding incoherently all the solutions:

$$
I(\rho, \emptyset)=\sum_{i=1}^{\mathrm{N}_{\mathrm{F}}}\left|E_{\mathrm{HP}}^{\mathrm{rcstr}}\left(\rho, \emptyset ; \mathrm{tx}_{\mathrm{m}} ; f_{i}\right)\right|^{2}
$$

Fig 9 shows the image of the head obtained after applying eq. (9) to the simulated data, while Fig 10 refers to the image of the phantom obtained after applying eq. (9) to the measured data. As highlighted in Fig 9 and 10, the stroke can be clearly detected. It should be pointed out that in Fig 9, artefact removal has been achieved by subtracting the electric field calculated for Ella with the stroke and the electric field calculated for Ella. Conversely, in Fig 10 artefact removal has been achieved by subtracting the two measurements obtained for the two transmitting positions.

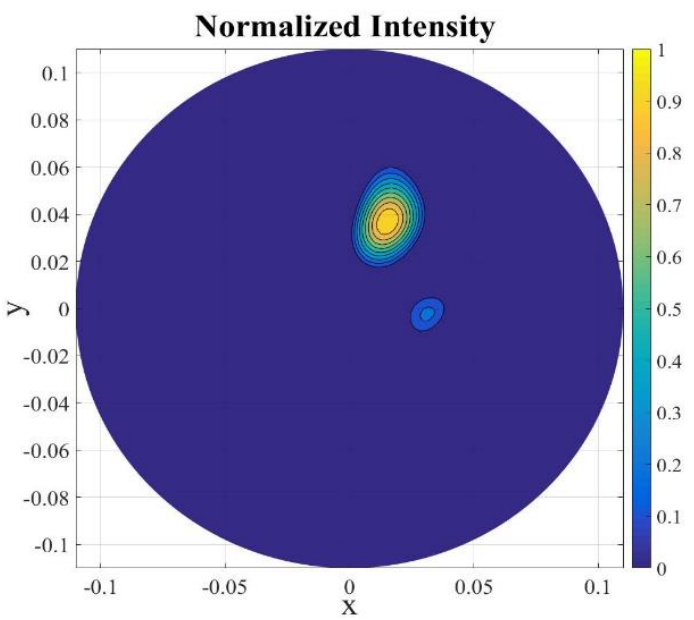

Fig 9. Imaging using the simulation data.

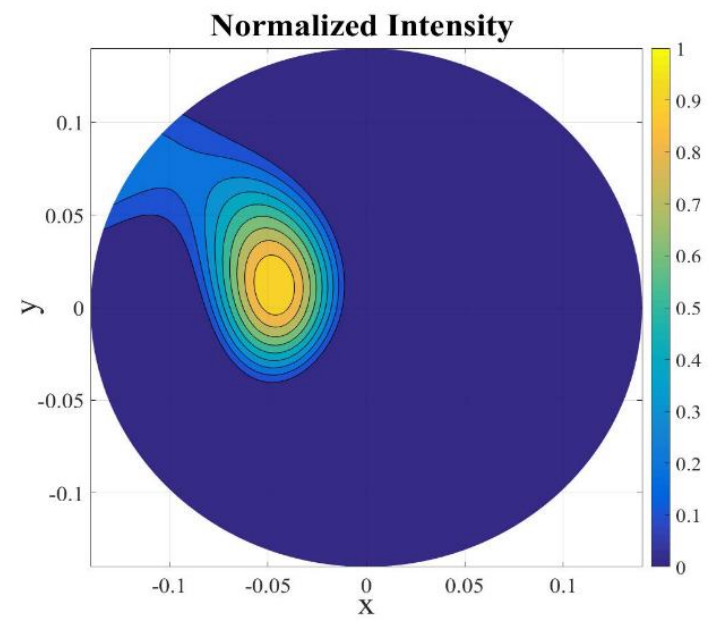

Fig 10. Imaging using the measurements data. 


\section{CONCLUSIONS}

In this paper, the development of a realistic human head phantom for haemorrhagic stroke detection has been described. We have fabricated the antennas and a phantom using dedicated recipes mimicking layers of Skull, grey matter and white matter and the blood. We have collected the signals with the same method in both simulations and measurements. We have presented both the simulation and measurement results. The simulation has been done to obtain the data all around the healthy head model and the head model with the haemorrhagic stroke with the aim of imaging. We employ here the subtraction between the difference of a healthy head and a head with stroke. As it is not realistic to do artefact removal between the subtraction of the measurement with a healthy head and the measurement with a head including the stroke, in a real measurement scenario, we will approach and show this difference by subtracting two nearby transmitter positions $\left(5^{\circ}\right.$ and $\left.0^{\circ}\right)$, as it has been done in the measurements performed in this paper. In measurements, we used the data collected from all around the phantom for imaging. This paper verifies that HP imaging can successfully be used to detect a haemorrhagic stroke inclusion in both a multilayer phantom and in simulations. The proposed antenna and the head phantom have been designed, simulated and fabricated, in order to image the human head. The ability to successfully apply HP to detect the haemorrhagic stroke inclusion in a multi-layered phantom has been verified. The phantom is then used for microwave imaging using the Huygens principle. Detection of the haemorrhagic stroke has been achieved after removing the artefacts, i.e. the images of the transmitter, through employing a subtraction between S21 obtained using measurements. Future research will be focused on the development of other phantoms that would consider other brain abnormalities, such as ischemic stroke and brain tumour, in order to image them.

\section{REFERENCES}

1. Feigin, V., "Stroke epidemiology in the developing world," The Lancet, Vol. 365, No. 9478, 2160-2161, Jun. 2005.

2. D. Ireland and M. Bialkowski, "Microwave head imaging for stroke detection," Progress In Electromagnetics Research M, Vol. 21, pp. 163175, 2011.

3. Ma, H., J. Ly, and G. A. Donnan, "TIA and stroke: A management guide for GPs," Medicine Today, Vol. 7, No. 5, May 2006.

4. Libman, R. B., E. Wirkowski, J. Alvir, and T. H. Rao, "Conditions that mimic stroke in the emergency department. Implications for acute stroke trials," Archives of Neurology, 1119-1122, 1995.

5. Ireland, D. and M. E. Bialkowski, "Feasibility study on microwave stroke detection using a realistic phantom and the FDTD method," Asia Pacific Microwave Conference, 1360\{1363, Yokohama, Japan, December 2010.

6. Serguei, Y., Y. Semenov, and D. R. Corfield, "Microwave tomography for brain imaging: Feasibility assessment for stroke detection," International Journal of Antennas and Propagation, Vol. 2008, Article ID 254830, 8, 2008, doi:10.1155/2008/254830.

7. Trefnam, H. and M. Persson, "Antenna array design for brain monitoring," Antennas and Propagation Society International Symposium, (AP-S 2008), San Diego, Ca, Jul. 2008.

8. E. M. Staderini, "UWB radars in medicine", in IEEE Aerospace and Electronic Systems Magazine, vol. 17, no. 1, pp. 13-18, Jan. 2002.

9. D. Ireland and M. Bialkowski, "Microwave head imaging for stroke detection," Progress In Electromagnetics Research M, Vol. 21, pp. 163175, 2011.

10. N. Ghavami, G. Tiberi, D. J. Edwards and A. Monorchio, "UWB Microwave Imaging of Objects With Canonical Shape," IEEE Transactions on Antennas and Propagation, vol. 60, no. 1, pp. 231-239, Jan. 2012.

11. N. Seladji-Hassaine and F. T. Bendimerad, "Compact Triangular Microstrip Antenna with Fractal Ground," Proc. Academics World 10th International Conference, Istanbul, Turkey, Dec. 2015.

12. L. Chandra Paul and N. Sultan, "Design, Simulation and Performance Analysis of a Line Feed Rectangular Micro-Strip Patch Antenna," International Journal of Engineering Sciences \& Emerging Technologies, vol. 4, Issue. 2, pp. 117-126, Feb. 2013.

13. M. Mathur, A. Vats and A. Agarwal, "A new design formulae for feed line dimensions of the rectangular microstrip patch antenna by using equivalent design concept," 2015 International Conference on Signal Processing and Communication (ICSC), Noida, 2015, pp. 105-110.

14. C. A. Balanis, "Antenna Theory: Analysis and Design”, Second Edition, New York, John Wiley \& Sons, Inc., 1997. 
15. Z. A. Abed Al-Husain, S. K. Ahmed and A. A. Osman, "Design of Ultrawideband micrstrip antennas with slots and fractal based ground plane", Journal of Al Rafidain University College, Issue No. 34, pp. 244-258, 2014.

16. C. Gabriel, "Compilation of the Dielectric Properties of Body Tissues at RF and Microwave Frequencies”, Report N.AL/OE-TR- 1996-0037, Occupational and environmental health directorate, Radiofrequency Radiation Division, Brooks Air Force Base, Texas (USA), 1996.

17. M. Aldhaeebi and I. Elshafiey, "New Antenna Design for Hyperthermia Treatment of Human Head," 2014 UKSim-AMSS 16th International Conference on Computer Modelling and Simulation, Cambridge, 2014, pp. 96-100.

18. https://itis.swiss/virtual-population/tissue-properties/database/dielectric-properties/

19. S. Symeonidis, W. G. Whittow and C. Panagamuwa, "Design and characterization of a three material anatomical bone phantom for implanted antenna applications", Loughborough Antennas \& Propagation Conference (LAPC 2017), Loughborough, 2017, pp. 1-5.

20. P. M. Meaney, C. J. Fox, S. D. Geimer and K. D. Paulsen, "Electrical Characterization of Glycerin: Water Mixtures: Implications for Use as a Coupling Medium in Microwave Tomography", in IEEE Transactions on Microwave Theory and Techniques, vol. 65, no. 5, pp. 1471-1478, May 2017.

21. N. Ghavami, PP. Smith, G. Tiberi, D. Edwards, I. Craddock "Non-Iterative beamforming based on Huygens principle for multistatic ultrawide band radar: application to breast imaging," IET Microwaves, Antennas \& Propagation, Sep 2015, Vol.9, Iss.12, pp.1233-1240. 